\title{
Organoamine-Directed Assembly of 5p-4f Heterometallic Cluster Substituted Polyoxometalates: Luminescence and Proton Conduction Properties
}

Hui-Ping Xiao, ${ }^{\dagger}$ Rong-Tao Zhang, ${ }^{\dagger}$ Zhong Li, $^{\dagger}$ Yu-Feng Xie,${ }^{\dagger}$ Min Wang, ${ }^{\dagger}$ Yi-Da Ye,${ }^{\dagger}$ Cai Sun,${ }^{\dagger}$ YanQiong Sun, ${ }^{\dagger}$ Xin-Xiong Li, ${ }^{* \dagger}$ Shou-Tian Zheng ${ }^{\dagger}$

$\dagger$ State Key Laboratory of Photocatalysis on Energy and Environment, College of Chemistry, Fuzhou University, Fuzhou, Fujian 350108, China

*State Key Laboratory of Structural Chemistry, Fujian Institute of Research on the Structure of Matter, and Graduate School of the Chinese Academy of Sciences, Fuzhou, Fujian 350002, China

\section{This file includes:}

Section 1: Additional structural figures $\quad$ S1-S2

Section 2: Additional characterizations $\quad$ S3-S6

Section 3: Additional tables $\quad$ S7-S9

$\begin{array}{lr}\text { Section 4: References } & \mathbf{S 1 0}\end{array}$ 


\section{Section 1: Additional structural figures}
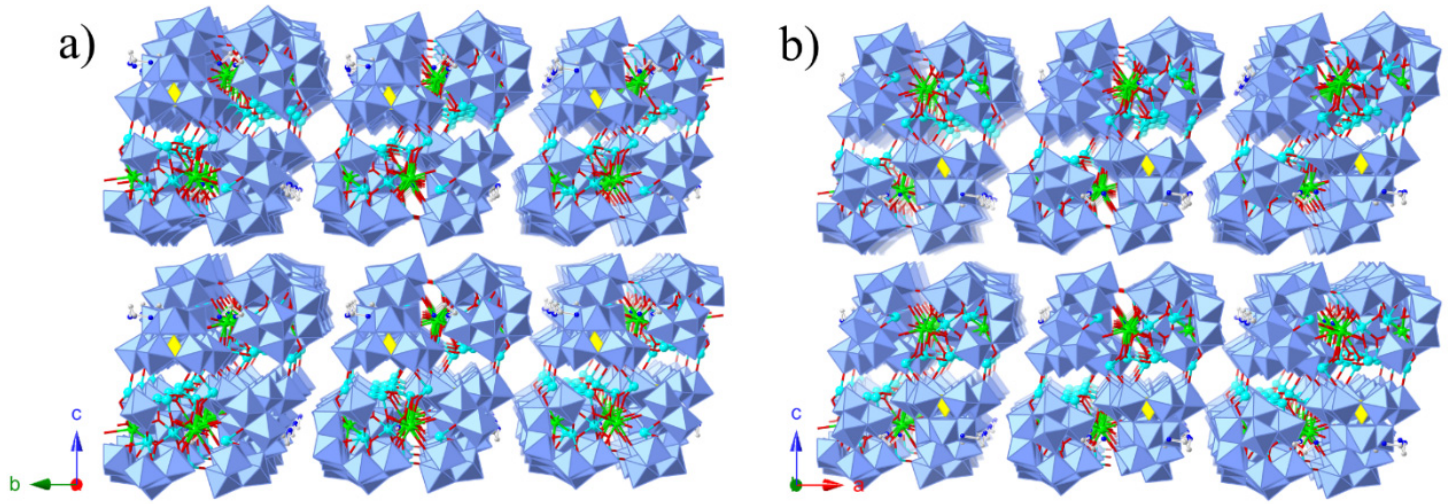

Figure S1 Packing arrangement of 1-Ce viewed a) along the $a$ axis and $\mathrm{b}$ ) along the $b$ axis.

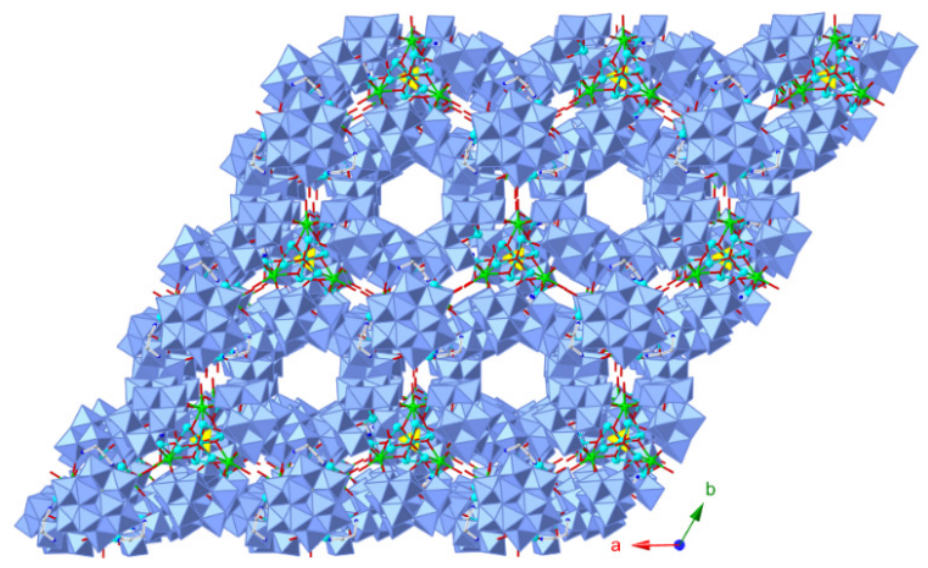

Figure S2 Packing arrangement of 1-Ce viewed along the $c$ axis.
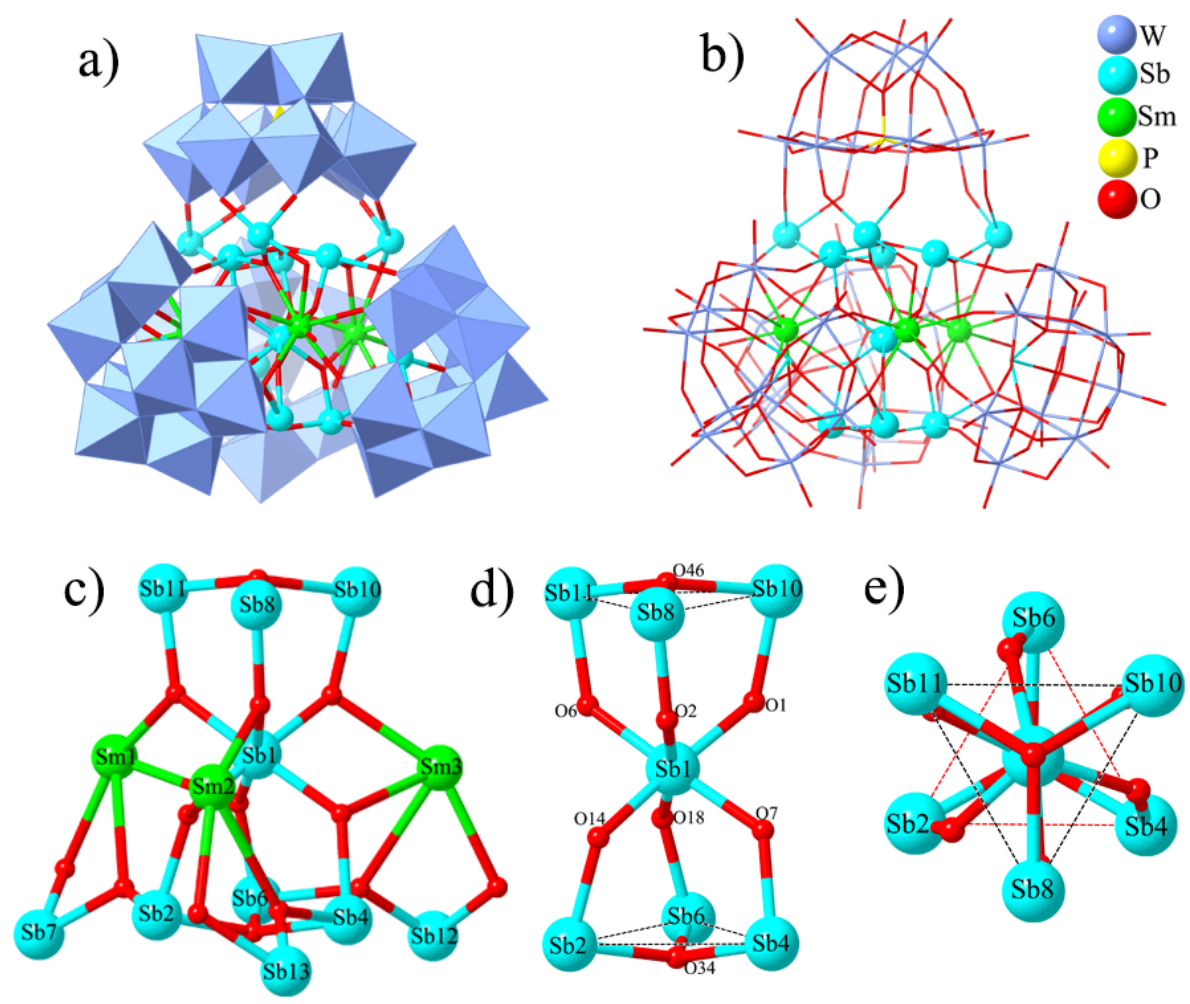

e)

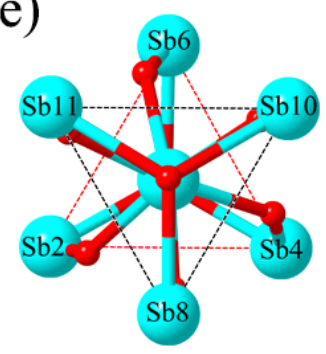


Figure S3 a) Polyhedral and stick representation of 2-Sm polyanion: $\left[\mathrm{Sb}^{\mathrm{III}}{ }_{9} \mathrm{Sb}^{\mathrm{V}} \mathrm{Sm}_{3} \mathrm{O}_{14}\left(\mathrm{H}_{2} \mathrm{O}\right)_{3}\right]\left[\left(\mathrm{SbW}_{9} \mathrm{O}_{33}\right)_{3}\left(\mathrm{PW}_{9} \mathrm{O}_{34}\right)\right]^{23-} ; \quad$ b) Combined wireframe/ball-and-stick representation of $\mathbf{2 - S m}$; c) Ball and stick representation of the $5 \mathrm{p}-4 \mathrm{f}$ heterometallic cluster $\left\{\mathrm{Sb}^{\mathrm{III}}{ }_{9} \mathrm{Sb}^{\mathrm{V}} \mathrm{Sm}_{3} \mathrm{O}_{14}\right\}$; d) Hourglass-shaped structure of $\left\{\mathrm{Sb}_{7} \mathrm{O}_{8}\right\}$ cluster; e) Top view of $\left\{\mathrm{Sb}_{7} \mathrm{O}_{8}\right\}$ cluster. Color codes: $\mathrm{WO}_{6}$, blue; $\mathrm{PO}_{4}$, yellow.

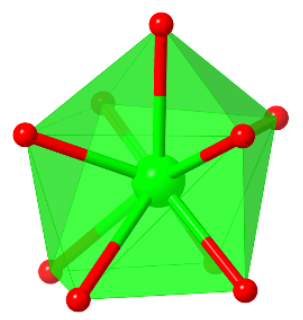

$\mathrm{Sm} 1$

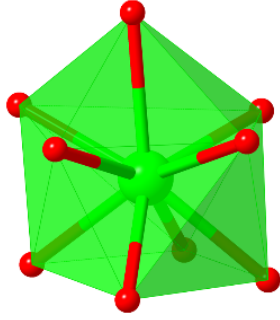

$\mathrm{Sm} 2$

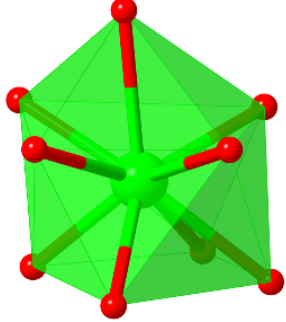

$\mathrm{Sm} 3$

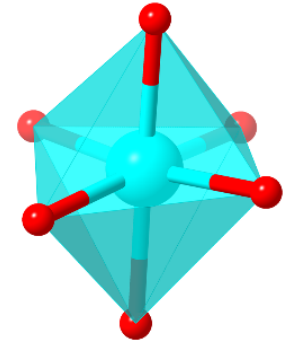

$\mathrm{Sb} 1$

Figure $\mathbf{S} 4$ View of the coordination environments of $\mathrm{Sm}^{3+}$ ions and $\mathrm{Sb}^{5+}$ ion in $\mathbf{1 - S m}$.

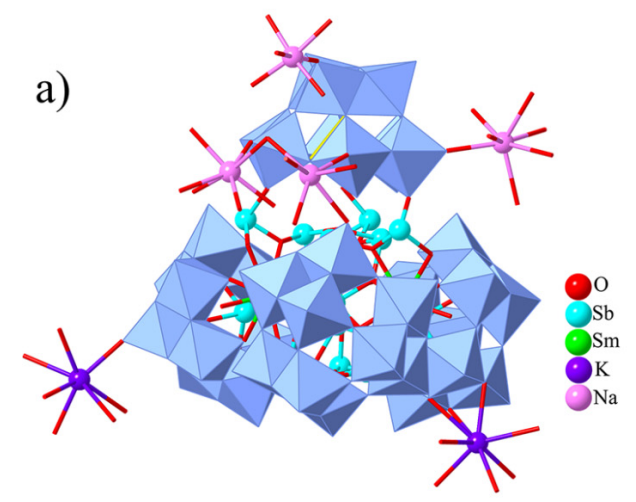

c)

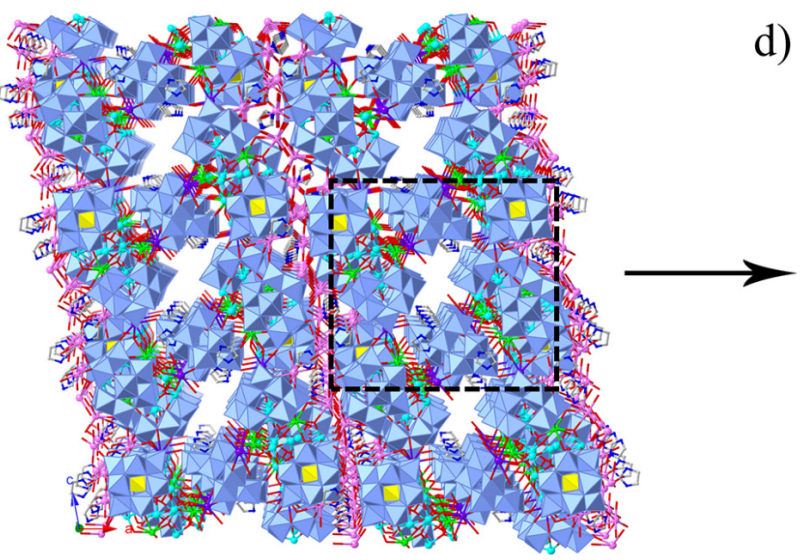

d) b)
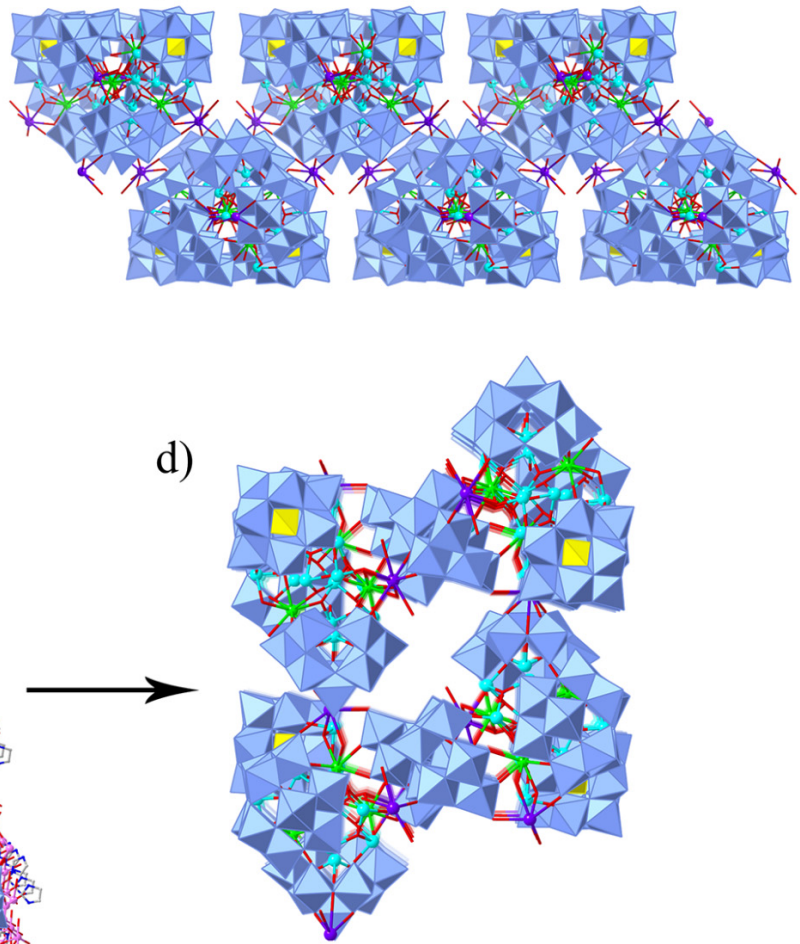

Figure S5 a) Polyhedral and stick representation of 2-Sm, c) and d) Views of the one dimensional "H"-shaped channel running along $c$ axis and $b$ axis. b) Packing arrangement of 2-Sm viewed along the $b$ axis. Color codes: $\mathrm{WO}_{6}$, blue; $\mathrm{PO}_{4}$, yellow. 


\section{Section 2: Characterizations}
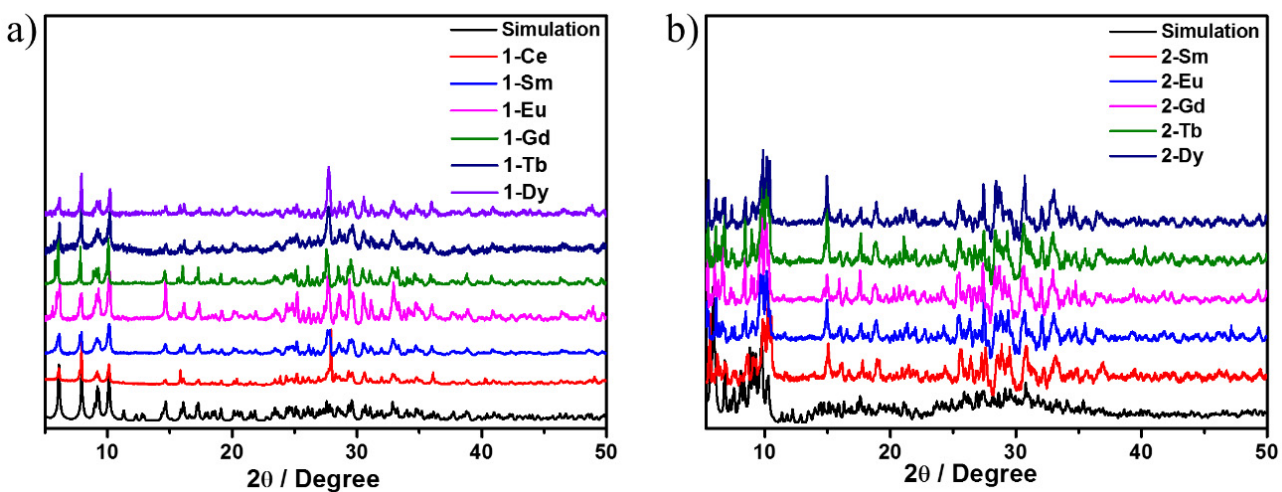

Figure S6 The simulated and experimental PXRD patterns of a) 1-Ln and b) 2-Ln.
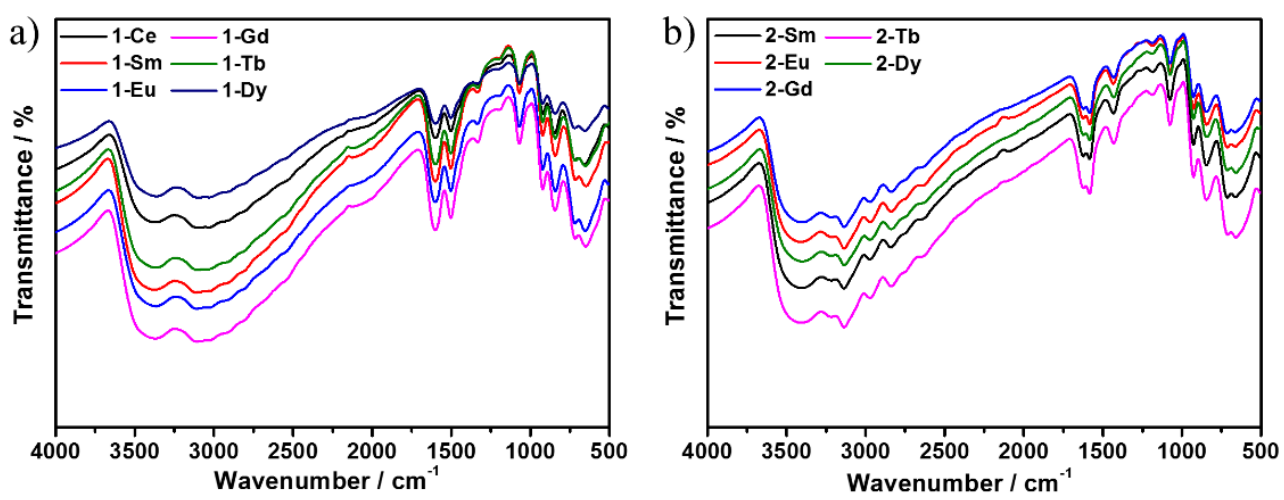

Figure S7 The IR spectra of a) 1-Ln and b) 2-Ln.
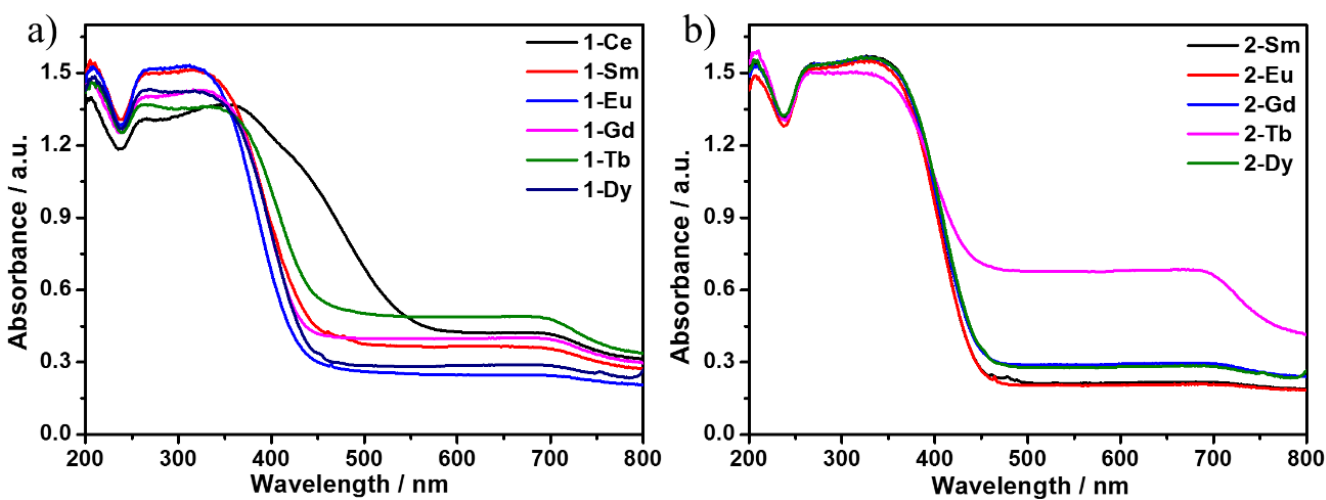

Figure S8 Solid-state UV-vis absorption spectra of a) 1-Ln and b) 2-Ln. 

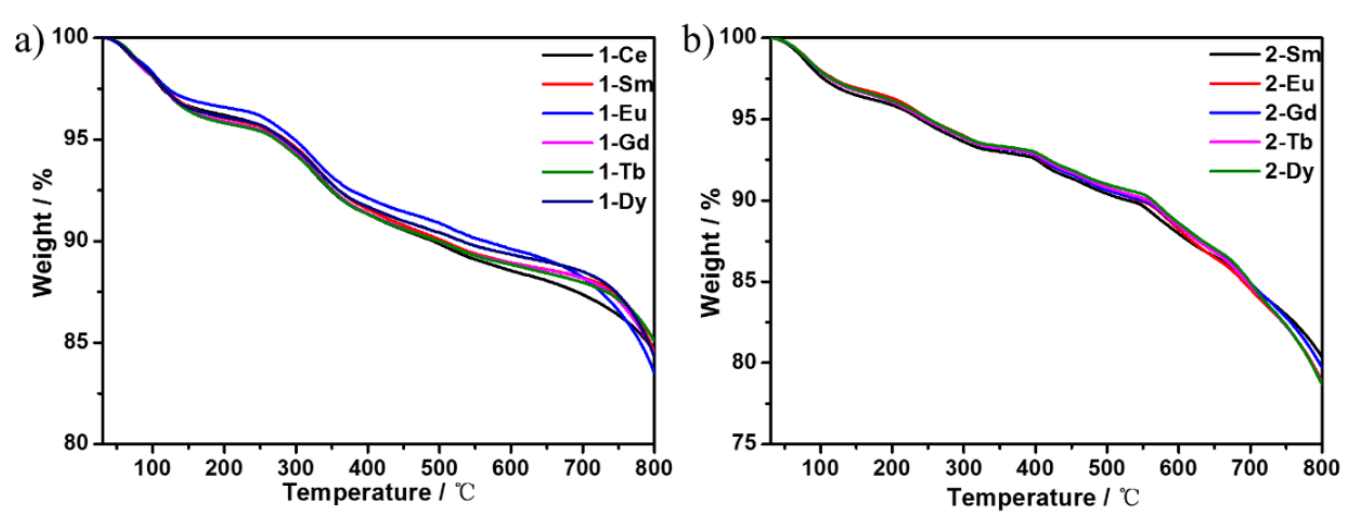

Figure S9 TG curves of a) 1-Ln and b) 2-Ln.

For the sake of investigating the thermal stability of $\mathbf{1 - L n}$ and 2-Ln, their TG analyses were performed in an air-flow atmosphere with a heating rate of $10{ }^{\circ} \mathrm{C} / \mathrm{min}$ at a temperature of $30-800{ }^{\circ} \mathrm{C}$. As shown in Figure S9a, their TG curves exhibit three continuous weight loss steps. The first weight loss of $\sim 4.2 \%\left(30-200{ }^{\circ} \mathrm{C}\right)$ is associated with the loss of c.a. 28 lattice water molecules, the second loss of $\sim 7.8 \%\left(200-700{ }^{\circ} \mathrm{C}\right)$ is due to coordinate water molecules and organic content $\left[\mathrm{H}_{2} \mathrm{en}\right]^{2+}$ degradation and the skeleton starts to decompose at $700^{\circ} \mathrm{C}$. As shown in Figure $\mathrm{S} 9 \mathrm{~b}$, their TG curves exhibit four continuous weight loss steps. The first weight loss of $\sim 3.8 \%\left(30-200{ }^{\circ} \mathrm{C}\right)$ is associated with the loss of c.a. 26 lattice water molecules, the second loss of $\sim 3.0 \%\left(200-400{ }^{\circ} \mathrm{C}\right)$ is due to coordinate water molecules, The third weight loss of $\sim 6.2 \%\left(30-650{ }^{\circ} \mathrm{C}\right)$ and organic content $[\mathrm{HIm}]^{+}$degradation, finally, the skeleton starts to decompose at $650^{\circ} \mathrm{C}$.
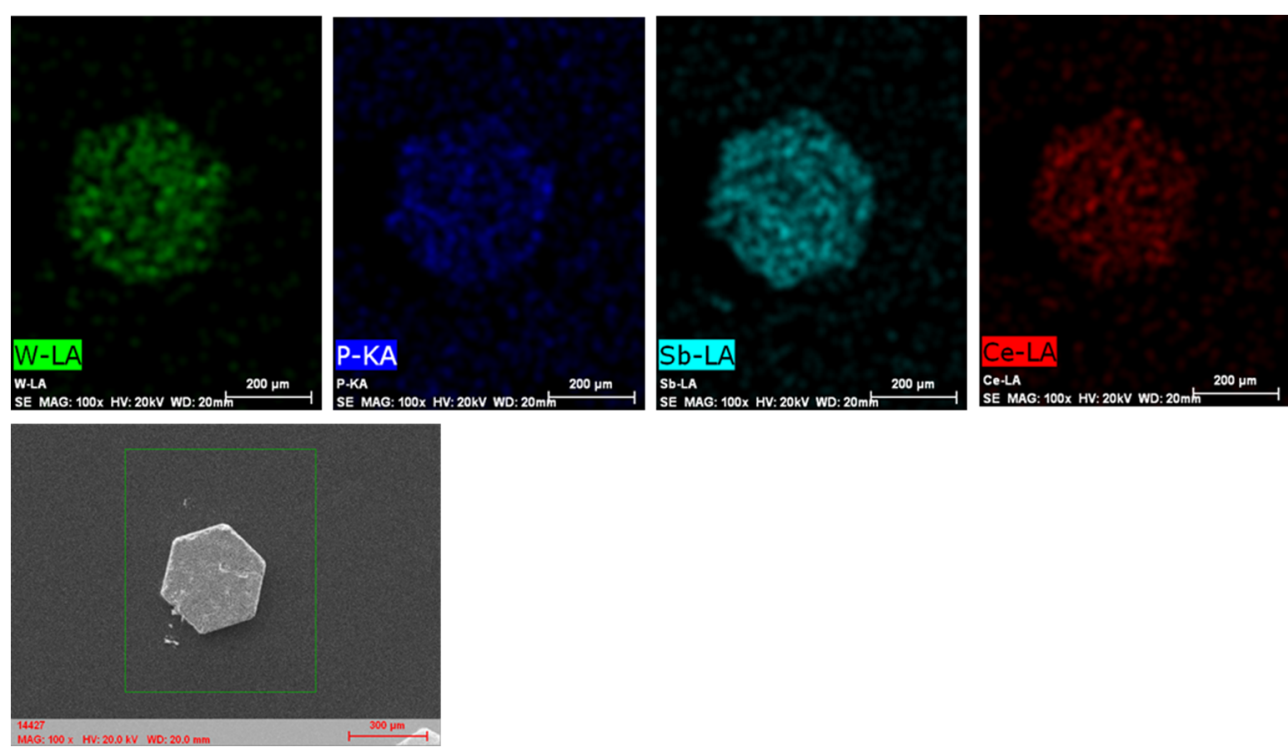

Figure S10 SEM images of 1-Ce with elemental maps for W, P, Sb and Ce. 

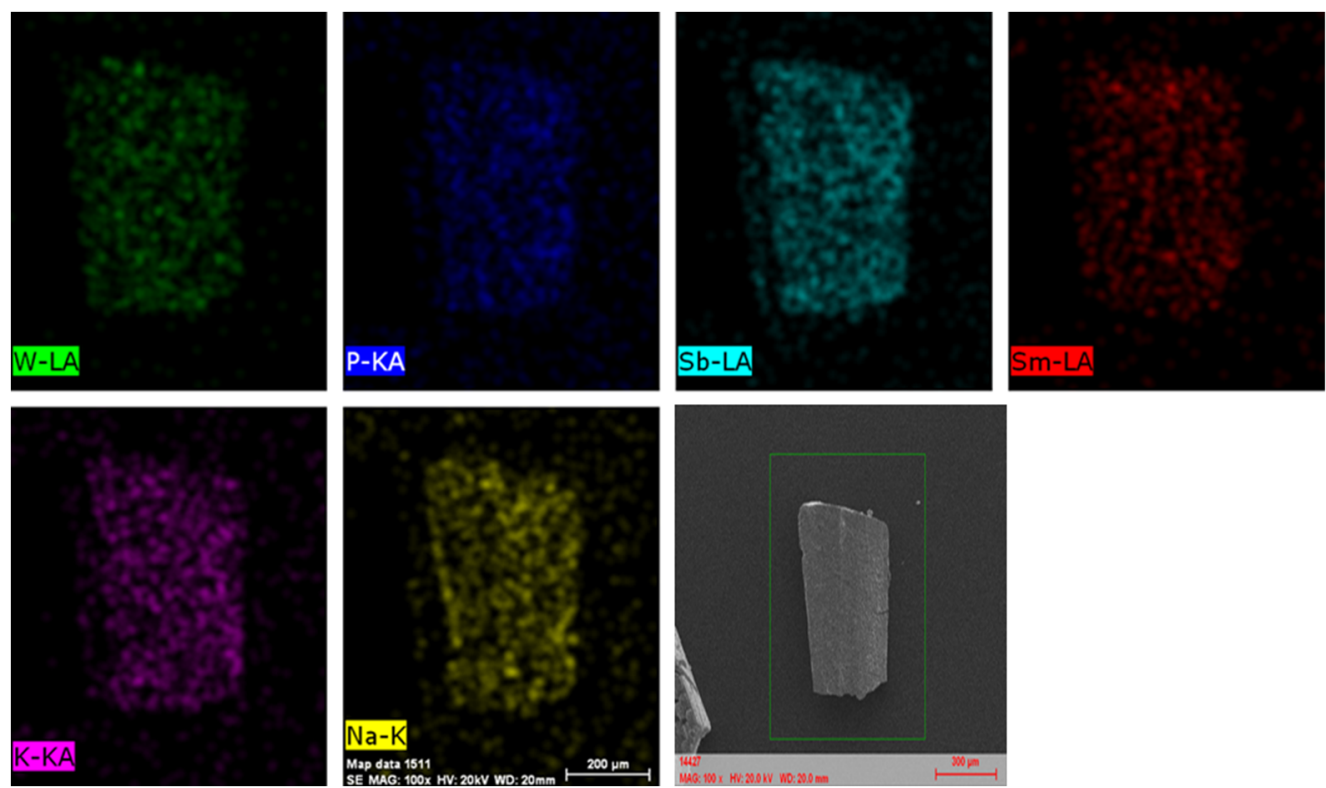

Figure S11 SEM images of 2-Sm with elemental maps for W, P, Sb, Sm, K and Na.

a)

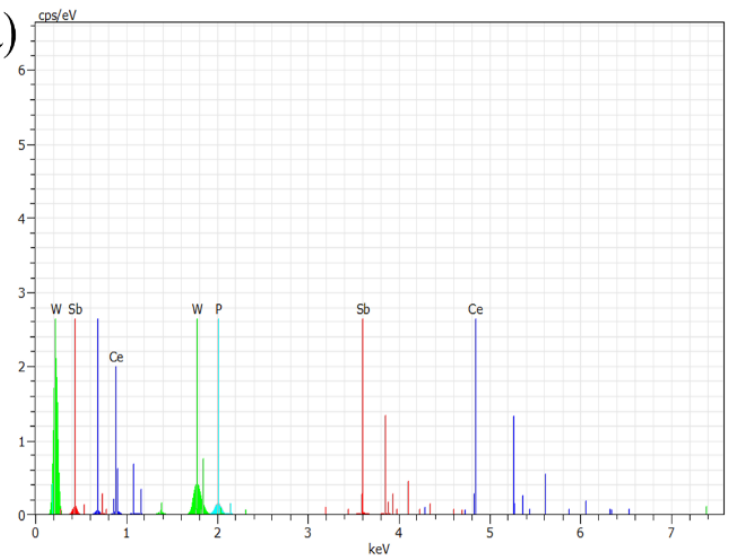

b)

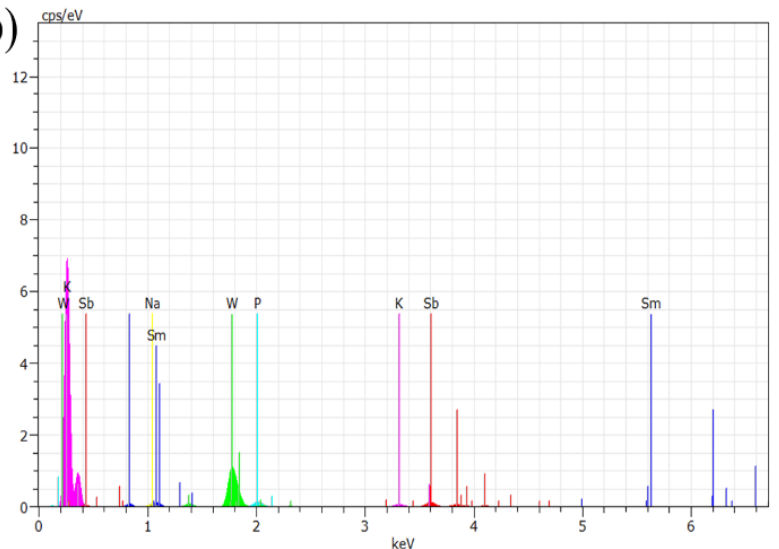

Figure S12 Energy-dispersive spectroscopy of a) 1-Ce and b) 2-Sm.

a)

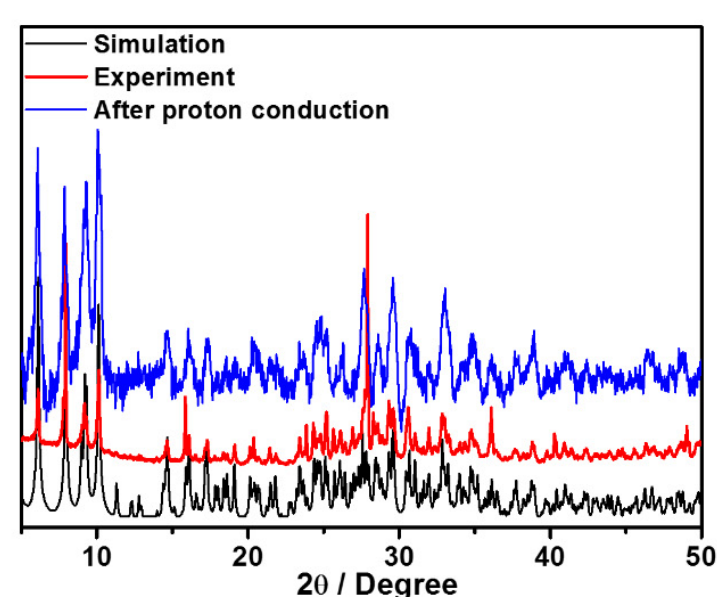

b)

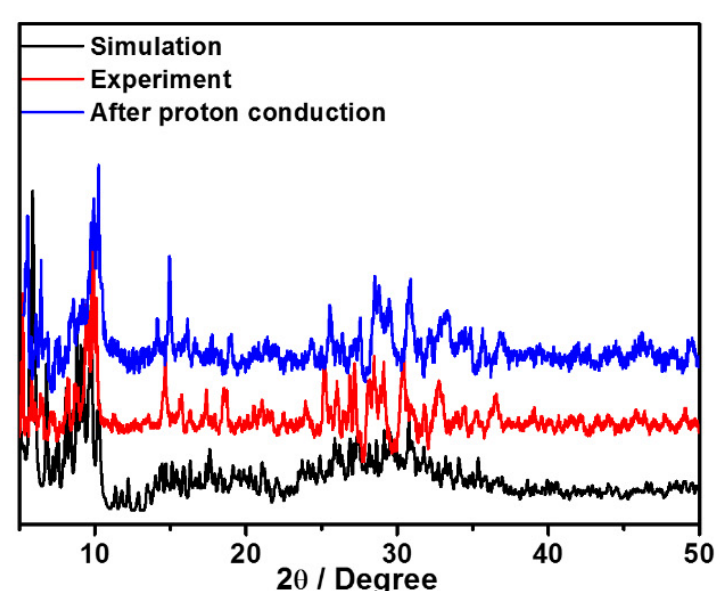

Figure S13 PXRD patterns after proton conduction for a) 1-Ce and b) 2-Sm. 

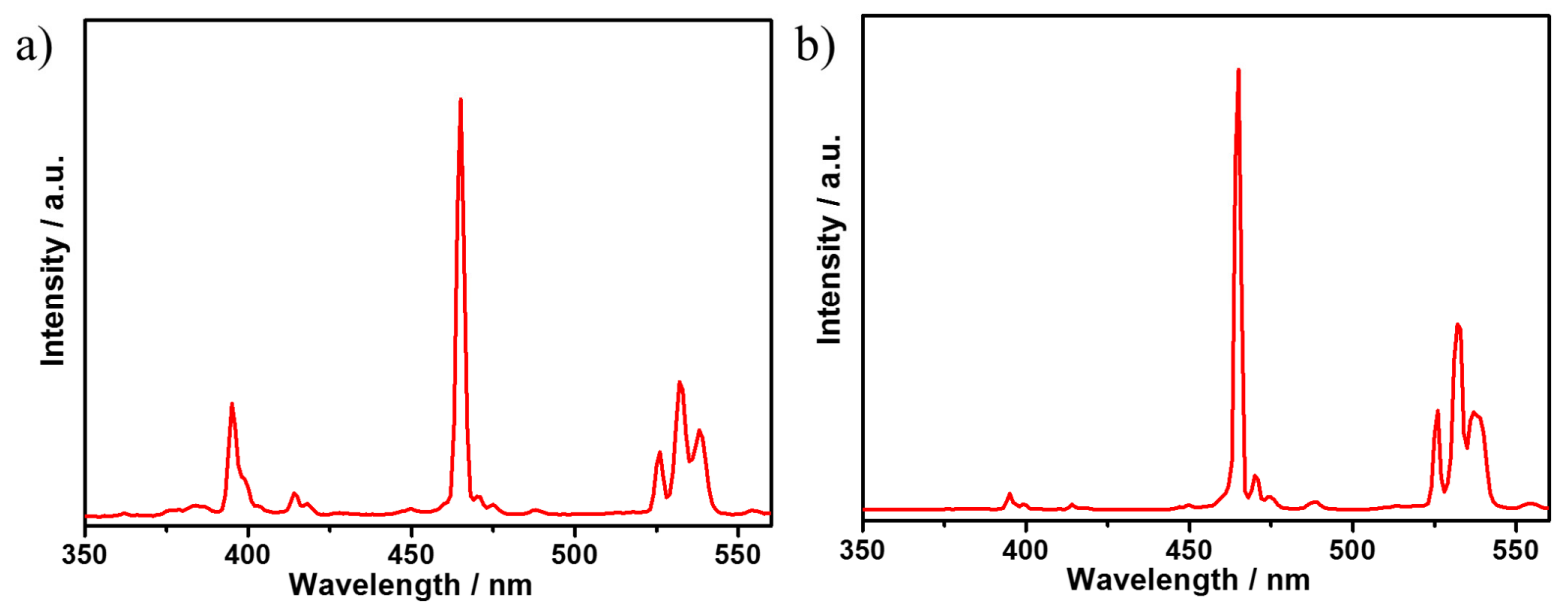

Figure S14 The excitation spectra of a) 1-Eu and b) 2-Eu.
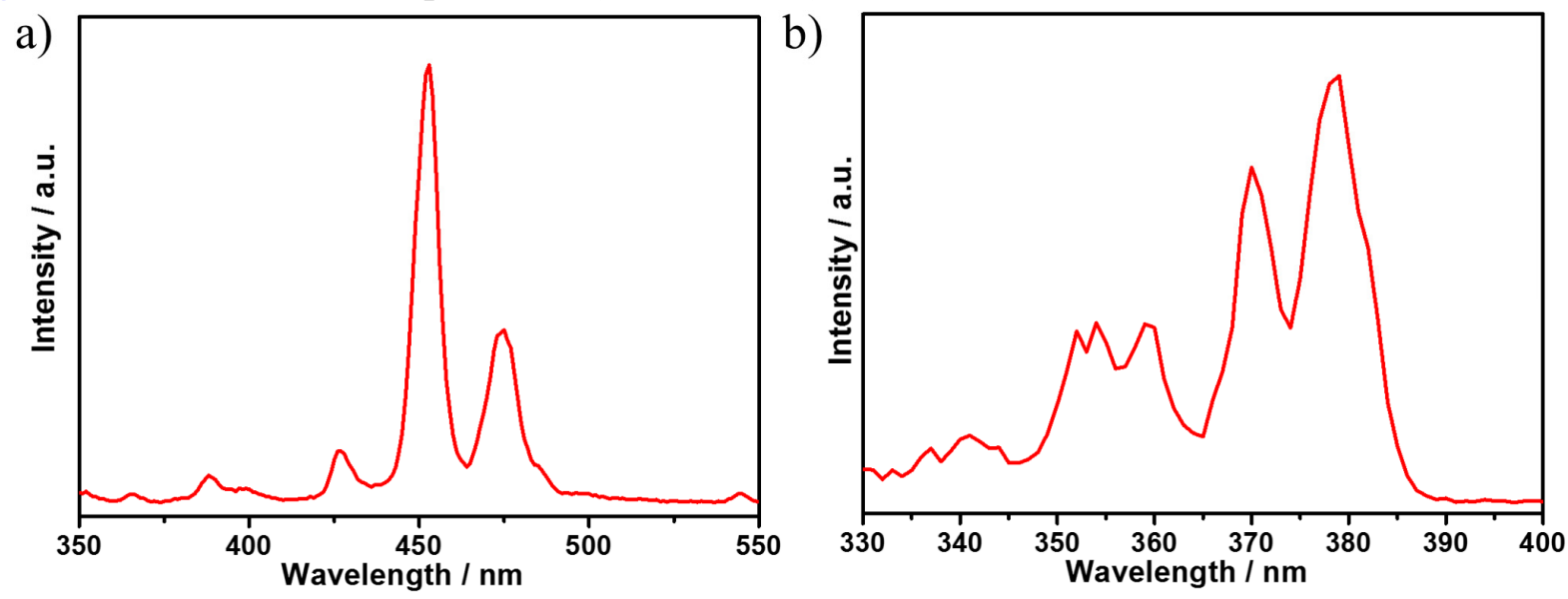

Figure S15 The excitation spectra of a) 2-Dy and b) 2-Tb. 
Section 3: Additional tables

Table S1 Bond valence sum (BVS) calculations of all W, Sm, P and Sb atoms in 1-Ce.

\begin{tabular}{cc}
\hline Atom & BVS \\
\hline $\mathrm{Ce}$ & 3.128 \\
$\mathrm{P}$ & 4.772 \\
$\mathrm{Sb}^{\mathrm{V}}$ & 5.104 \\
$\mathrm{Sb}^{\mathrm{III}}$ & $2.493-2.727$ \\
\hline
\end{tabular}

Table S2 Bond valence sum (BVS) calculations of all W, Sm, P and Sb atoms in 2-Sm.

\begin{tabular}{cc}
\hline Atom & BVS \\
\hline $\mathrm{Sm}$ & $2.991-3.159$ \\
$\mathrm{P}$ & 4.984 \\
$\mathrm{Sb}^{\mathrm{V}}$ & 5.108 \\
$\mathrm{Sb}^{\mathrm{III}}$ & $2.401-2.841$ \\
\hline
\end{tabular}

Table S3 Comparison of the conductivity of 1-Ce and 2-Sm with representative heteropolyoxometalates conducting materials

\begin{tabular}{|c|c|c|c|c|}
\hline Compounds & $\begin{array}{l}\text { Conductivity } \\
\qquad\left(\mathbf{S c m}^{-1}\right)\end{array}$ & $\begin{array}{l}\text { Temperature } \\
\left({ }^{\circ} \mathrm{C}\right)\end{array}$ & $\begin{array}{l}\text { Relative } \\
\text { humidity }\end{array}$ & Reference \\
\hline $\mathrm{H}_{17}\left(\mathrm{H}_{2} \mathrm{en}\right)_{3}\left[\mathrm{Sb}_{9}^{\mathrm{III}} \mathrm{Sb}^{\mathrm{V}} \mathrm{Ln}_{3} \mathrm{O}_{14}\left(\mathrm{H}_{2} \mathrm{O}\right)_{3}\right]\left[\left(\mathrm{SbW}_{9} \mathrm{O}_{33}\right)_{3}\left(\mathrm{PW}_{9} \mathrm{O}_{34}\right)\right] \cdot 28 \mathrm{H}_{2} \mathrm{O}$ & $1.64 \times 10^{-2}$ & 85 & $98 \%$ & This work \\
\hline $\mathrm{H}_{12}(\mathrm{HIm})_{4} \mathrm{~K}_{2} \mathrm{Na}_{4}\left(\mathrm{H}_{2} \mathrm{O}\right)_{9}\left[\mathrm{Sb}_{9}^{\mathrm{II}} \mathrm{Sb}^{\mathrm{V}} \mathrm{Ln}_{3} \mathrm{O}_{14}\left(\mathrm{H}_{2} \mathrm{O}\right)_{3}\right]\left[\left(\mathrm{SbW}_{9} \mathrm{O}_{33}\right)_{3}\left(\mathrm{PW}_{9} \mathrm{O}_{34}\right)\right] \cdot 26 \mathrm{H}_{2} \mathrm{O}$ & $4.57 \times 10^{-4}$ & 85 & $98 \%$ & This work \\
\hline $\mathrm{Na}_{17} \mathrm{~K}_{14}\left(\mathrm{H}_{2} \mathrm{pip}\right)_{10} \mathrm{H}_{8}\left[\mathrm{La}_{27} \mathrm{Ge}_{10} \mathrm{~W}_{106} \mathrm{O}_{406}(\mathrm{OH})_{4}\left(\mathrm{H}_{2} \mathrm{O}\right)_{24}\right] \cdot \mathrm{nH}_{2} \mathrm{O}(\mathrm{n} \approx 130)$ & $1.5 \times 10^{-2}$ & 85 & $98 \%$ & S1 \\
\hline$\left[\mathrm{La}_{3}\left(\mathrm{H}_{2} \mathrm{O}\right)_{22}\right]\left[\mathrm{P}_{2} \mathrm{~W}_{15} \mathrm{Ta}_{3} \mathrm{O}_{62}\right] \cdot \mathrm{nH}_{2} \mathrm{O}$ & $1.26 \times 10^{-2}$ & 85 & $98 \%$ & $\mathrm{~S} 2$ \\
\hline $\mathrm{Na}_{2}\left[\mathrm{Gd}_{2}\left(\mathrm{H}_{2} \mathrm{O}\right)_{11}\right]_{2}\left[\mathrm{Gd}_{3}\left(\mathrm{H}_{2} \mathrm{O}\right)_{2}-\left(\alpha-\mathrm{SiW}_{11} \mathrm{O}_{39}\right)_{2}\right]_{2} \cdot 69 \mathrm{H}_{2} \mathrm{O}$ & $3.54 \times 10^{-3}$ & 85 & $98 \%$ & $\mathrm{~S} 3$ \\
\hline$\left\{\left[\mathrm{W}_{14} \mathrm{Ce}^{\mathrm{IV}}{ }_{6} \mathrm{O}_{61}\right]\left(\left[\mathrm{W}_{3} \mathrm{Bi}_{6} \mathrm{Ce}_{3}^{\mathrm{III}}\left(\mathrm{H}_{2} \mathrm{O}\right)_{3} \mathrm{O}_{14}\right]\left[\mathrm{B}-\alpha-\mathrm{BiW}_{9} \mathrm{O}_{33}\right]_{3}\right)_{2}\right\}^{34-}$ & $2.4 \times 10^{-3}$ & 25 & $90 \%$ & S4 \\
\hline $\mathrm{H}_{9}\left[\mathrm{La}_{9} \mathrm{~W}_{8}\left(\mu_{4}-\mathrm{O}\right)_{12}\left(\mu_{2}-\mathrm{O}\right)_{24}\left(\mathrm{H}_{2} \mathrm{O}\right)_{24}\right]\left(\mathrm{SiW}_{12} \mathrm{O}_{40}\right)_{3} \cdot 60 \mathrm{H}_{2} \mathrm{O}$ & $4.75 \times 10^{-4}$ & 85 & $98 \%$ & S5 \\
\hline $\begin{array}{l}\mathrm{Na}_{4} \mathrm{~K}_{4}\left(\mathrm{H}_{2} \mathrm{pip}\right)_{6}\left(\mathrm{H}_{2} \mathrm{O}\right)_{10}\left\{\mathrm{La}_{10}\left(\mu_{3}-\mathrm{OH}\right)_{2}\left(\mathrm{H}_{2} \mathrm{O}\right) 10\left[\alpha(1,8)-\mathrm{GeW}_{10} \mathrm{O}_{38}\right]_{2}[\beta(4,11)-\right. \\
\left.\left.\mathrm{GeW}_{10} \mathrm{O}_{38}\right]_{2}\right\} \cdot 29 \mathrm{H}_{2} \mathrm{O}\end{array}$ & $6.35 \times 10^{-4}$ & 85 & $98 \%$ & S6 \\
\hline$\left[\mathrm{Ce}^{\mathrm{III}}\left(\mathrm{H}_{2} \mathrm{O}\right)_{6}\right]\left\{\left[\mathrm{Ce}^{\mathrm{IV}}{ }_{7} \mathrm{Ce}_{3} \mathrm{III}_{3} \mathrm{O}_{6}(\mathrm{OH})_{6}\left(\mathrm{CO}_{3}\right)\left(\mathrm{H}_{2} \mathrm{O}\right)_{11}\right]\left[\left(\mathrm{P}_{2} \mathrm{~W}_{16} \mathrm{O}_{59}\right)\right]_{3}\right\}^{7}$ & $2.65 \times 10^{-4}$ & 100 & $75 \%$ & S7 \\
\hline$\left[\mathrm{H}_{2} \mathrm{en}\right]_{4}\left[\mathrm{Ni}_{5}(\mathrm{OH})_{3}(\operatorname{trzS})_{3}(\mathrm{en})\left(\mathrm{H}_{2} \mathrm{O}\right)\left(\mathrm{B}-\alpha-\mathrm{PW}_{9} \mathrm{O}_{34}\right)\right] \cdot 6 \mathrm{H}_{2} \mathrm{O}$ & $1.3 \times 10^{-5}$ & 85 & $98 \%$ & S8 \\
\hline$\left[\mathrm{Cu}_{3}\left(\mu_{3}-\mathrm{OH}\right)\left(\mathrm{H}_{2} \mathrm{O}\right)_{3}(\text { atz })_{3}\right]_{3}\left[\mathrm{P}_{2} \mathrm{~W}_{18} \mathrm{O}_{62}\right] \cdot 14 \mathrm{H}_{2} \mathrm{O}$ & $4.4 \times 10^{-6}$ & 25 & $97 \%$ & S9 \\
\hline $\mathrm{Cu}_{6}(\mathrm{Trz})_{10}\left(\mathrm{H}_{2} \mathrm{O}\right)_{4}\left[\mathrm{H}_{2} \mathrm{SiW}_{12} \mathrm{O}_{40}\right] \cdot 8 \mathrm{H}_{2} \mathrm{O}$ & $1.84 \times 10^{-6}$ & 95 & $95 \%$ & S10 \\
\hline
\end{tabular}


Table S4 Selected bond lengths ( $\AA$ ) for 1-Ce

\begin{tabular}{lllllllll}
\hline Atom 1 & Atom 2 & Distance/ $\mathbf{\AA}$ & Atom 1 & Atom 2 & Distance/ $\AA$ & Atom 1 & Atom 2 & Distance/ $\mathbf{A}$ \\
\hline SB1 & O50 & 1.9742 & SB3 & O42 & 2.0885 & P1 & O46 & 1.5461 \\
SB1 & O7 & 1.9972 & SB3 & O11 & 2.5916 & P1 & O46 & 1.5461 \\
SB1 & O10 & 2.0032 & SB4 & O8 & 1.9524 & P1 & O15 & 1.592 \\
SB2 & O9 & 1.9723 & SB4 & O14 & 2.0025 & CE1 & O23 & 2.3989 \\
SB2 & O9 & 1.9723 & SB4 & O52 & 2.0089 & CE1 & O13 & 2.4399 \\
SB2 & O9 & 1.9723 & SB4 & O5 & 2.6854 & CE1 & O37 & 2.4736 \\
SB2 & O6 & 1.9885 & SB5 & O9 & 1.9416 & CE1 & O9 & 2.5159 \\
SB2 & O6 & 1.9885 & SB5 & O21 & 2.0232 & CE1 & O51 & 2.5479 \\
SB2 & O6 & 1.9885 & SB5 & O28 & 2.1224 & CE1 & O29 & 2.5684 \\
SB3 & O6 & 1.9535 & SB5 & O51 & 2.4271 & CE1 & O6 & 2.6145 \\
SB3 & O8 & 1.9686 & P1 & O46 & 1.5461 & CE1 & O52 & 2.6233 \\
\hline
\end{tabular}

Table S5 Selected bond lengths ( $\AA$ ) for 2-Sm

\begin{tabular}{|c|c|c|c|c|c|c|c|c|}
\hline Atom 1 & Atom 2 & Distance/ $/ \AA$ & Atom 1 & Atom 2 & Distance/Å & Atom 1 & Atom 2 & Distance/Å \\
\hline SB1 & $\mathrm{O} 1$ & 1.9494 & SB7 & $\mathrm{O} 43$ & 2.4408 & $\mathrm{P} 1$ & $\mathrm{O} 32$ & 1.5383 \\
\hline SB1 & O14 & 1.9677 & SB8 & $\mathrm{O} 2$ & 1.9427 & $\mathrm{P} 1$ & O88 & 1.6029 \\
\hline SB1 & $\mathrm{O} 7$ & 1.9831 & SB8 & O138 & 2.0124 & SM1 & O52 & 2.3586 \\
\hline SB1 & O6 & 1.9842 & SB8 & O46 & 2.0841 & SM1 & O50 & 2.3735 \\
\hline SB1 & O18 & 1.9944 & SB8 & O93 & 2.339 & SM1 & $\mathrm{O} 75$ & 2.3742 \\
\hline SB1 & $\mathrm{O} 2$ & 2.002 & SB9 & O49 & 1.9941 & SM1 & O6 & 2.4358 \\
\hline SB2 & $\mathrm{O} 30$ & 1.9591 & SB9 & O87 & 2.0073 & SM1 & O157 & 2.509 \\
\hline SB2 & $\mathrm{O} 14$ & 1.9776 & SB9 & O109 & 2.0233 & SM1 & O66 & 2.5391 \\
\hline SB2 & $\mathrm{O} 34$ & 2.0785 & SB10 & $\mathrm{O} 1$ & 1.9769 & SM1 & $\mathrm{O} 18$ & 2.5634 \\
\hline SB2 & $\mathrm{O} 123$ & 2.5752 & SB10 & $\mathrm{O} 37$ & 1.9984 & SM1 & $\mathrm{O} 28$ & 2.6084 \\
\hline SB3 & O102 & 1.9609 & SB10 & O46 & 2.1265 & SM2 & O67 & 2.3514 \\
\hline SB3 & O5 & 1.9875 & SB10 & $\mathrm{O} 12$ & 2.3517 & SM2 & $\mathrm{O} 15$ & 2.3797 \\
\hline SB3 & $\mathrm{O} 13$ & 1.997 & SB11 & O6 & 1.9758 & SM2 & O126 & 2.4369 \\
\hline SB4 & $\mathrm{O} 33$ & 1.9939 & SB11 & O8 & 2.0213 & SM2 & $\mathrm{O} 1$ & 2.456 \\
\hline SB4 & $\mathrm{O} 7$ & 1.9974 & SB11 & O46 & 2.105 & SM2 & O156 & 2.526 \\
\hline SB4 & $\mathrm{O} 34$ & 2.0739 & SB11 & $\mathrm{O} 28$ & 2.3519 & SM2 & O57 & 2.5282 \\
\hline SB4 & $\mathrm{O} 24$ & 2.5471 & SB12 & $\mathrm{O} 70$ & 1.9545 & SM2 & $\mathrm{O} 7$ & 2.541 \\
\hline SB5 & O59 & 1.9527 & SB12 & O69 & 2.0028 & SM2 & $\mathrm{O} 12$ & 2.6273 \\
\hline SB5 & O74 & 1.9801 & SB12 & O57 & 2.0467 & SM3 & O62 & 2.3289 \\
\hline SB5 & O35 & 1.9802 & SB12 & O81 & 2.4401 & SM3 & $\mathrm{O} 78$ & 2.3397 \\
\hline SB6 & O18 & 1.9506 & SB13 & $\mathrm{O} 33$ & 1.9427 & SM3 & $\mathrm{O} 40$ & 2.3768 \\
\hline SB6 & O70 & 1.9883 & SB13 & O29 & 2.015 & SM3 & $\mathrm{O} 2$ & 2.4223 \\
\hline SB6 & O34 & 2.1303 & SB13 & O103 & 2.021 & SM3 & O29 & 2.5228 \\
\hline SB6 & O137 & 2.4925 & SB13 & O56 & 2.4181 & SM3 & $\mathrm{O} 14$ & 2.5488 \\
\hline SB7 & O116 & 1.9567 & $\mathrm{P} 1$ & O91 & 1.5095 & SM3 & O158 & 2.5646 \\
\hline
\end{tabular}




\begin{tabular}{lllllllll}
\hline SB7 & O30 & 1.9921 & P1 & O48 & 1.5185 & SM3 & O93 & 2.605 \\
SB7 & O66 & 2.0688 & & & & & & \\
\hline
\end{tabular}




\section{Section 4: References}

S1. Li, Z.; Li, X. X.; Yang, T.; Cai, Z. W.; Zheng, S. T. Four-Shell Polyoxometalates Featuring High-Nuclearity Ln 26 Clusters: Structural Transformations of Nanoclusters into Frameworks Triggered by Transition-Metal Ions. Angew. Chem. Int. Ed. 2017, 56, 2664-2669.

S2. Peng, Q.; Li, S.; Wang, R.; Liu, S.; Xie, L.; Zhai, J.; Zhang, J.; Zhao, Q.; Chen, X. Lanthanide derivatives of Ta/W mixed-addendum POMs as proton-conducting materials. Dalton Trans. 2017, 46, 4157-4160.

S3. Liu, J. H.; Zhang, R. T.; Zhang, J.; Zhao, D.; Li, X. X.; Sun, Y. Q.; Zheng, S. T. A Series of 3D Porous LanthanideSubstituted Polyoxometalate Frameworks Based on Rare Hexadecahedral $\left\{\mathrm{Ln}_{6} \mathrm{~W}_{8} \mathrm{O}_{28}\right\}$ Heterometallic Cage-Shaped Clusters. Inorg. Chem. 2019, 58, 14734-14740.

S4. Liu, J. C.; Han, Q.; Chen, L. J.; Zhao, J. W.; Streb, C.; Song, Y. F. Aggregation of Giant Cerium-Bismuth Tungstate Clusters into a 3D Porous Framework with High Proton Conductivity. Angew. Chem. Int. Ed. 2018, 57, 8416-8420.

S5. Liu, J. H.; Lin, L. D.; Wang, G. Q.; Li, L. Y.; Sun, Y. Q.; Li, X. X.; Zheng, S. T. All-inorganic open frameworks based on gigantic four-shellLn@W $\mathrm{W}_{8} @ \mathrm{Ln}_{8} @\left(\mathrm{SiW}_{12}\right)_{6}$ clusters.Chem. Commun. 2020, 56, 10305-10308.

S6. Hao, Y.; Zhong, L.; Li, H.-H.; Zheng, S.-T. Two Lanthanide-Substituted Polyoxometalates Featuring Novel Crescent-Shaped Ln5 Clusters: Structures, Ion Conductivities, and Magnetic Properties. Cryst. Growth Des. 2019, 19, 1329-1335.

S7. Ma, P.; Wan, R.; Wang, Y.; Hu, F.; Zhang, D.; Niu, J.; Wang, J. Coordination-Driven Self-Assembly of a 2D GraphiteLike Framework Constructed from High-Nuclear $\mathrm{Ce}_{10}$ Cluster Encapsulated Polyoxotungstates. Inorg. Chem. 2016, $55,918-924$.

S8. Cao, G. J.; Liu, J. D.; Zhuang, T. T.; Cai, X. H.; Zheng, S. T. A polyoxometalate-organic supramolecular nanotube with high chemical stability and proton-conducting properties. Chem. Commun. 2015, 51, 2048-2051.

S9. Jiao, Y. Q.; Zang, H. Y.; Wang, X. L.; Zhou, E. L.; Song, B. Q.; Wang, C. G.; Shao, K. Z.; Su, Z. M. Self-assembled arrays of polyoxometalate-based metal-organic nanotubes for proton conduction and magnetism. Chem. Commun. 2015, 51, 11313-11316.

S10. Zhou, E. L.; Qin, C.; Huang, P.; Wang, X. L.; Chen, W. C.; Shao, K. Z.; Su, Z. M. A Stable Polyoxometalate-Pillared Metal-Organic Framework for Proton-Conducting and Colorimetric Biosensing. Chem. Eur. J. 2015, 21, 1189411898. 